\title{
Divertículo de Meckel en asociación a onfalocele y tetralogía de Fallot: reporte de caso y revisión de la literatura
}

\author{
Meckel's diverticulum in association with omphalocele and tetralogy of \\ Fallot: Case report and literature review
} \author{
Luis Felipe Cabrera ${ }^{3} \mathbb{D}$, Manuela Rodríguez ${ }^{4}$, Santiago Piñeros ${ }^{5}$ (D) \\ 1 Médico, residente de Cirugía general, Universidad El Bosque, Bogotá, D.C., Colombia. \\ 2 Médico, especialista en Cirugía pediátrica, Hospital Simón Bolívar, Bogotá, D.C., Colombia. \\ 3 Médico, especialista en Cirugía general, Universidad El Bosque, Bogotá, D.C., Colombia. \\ 4 Médico interno, Universidad de La Sabana, Chía, Colombia. \\ 5 Médico interno, Universidad El Bosque, Bogotá, D.C., Colombia.
}

Álvaro Eduardo Sánchez-Hernández $\mathbb{D}$, German Mogollón², Pablo Luengas² (D), Sergio Del Rio², Marcos Rotterman², Nicolás Mora², Sergio Rueda², Diana Alejandra Holguín²,

\section{Resumen}

El onfalocele o exónfalos se definen como un defecto congénito de la pared abdominal, que consiste en la herniación de las vísceras abdominales a través del anillo umbilical. Esta entidad rara vez se asocia a la comunicación del divertículo de Meckel con el saco del onfalocele. Teniendo en cuenta la escasa prevalencia de dicha entidad, compartimos el reporte de caso de un paciente recién nacido, con diagnóstico de onfalocele menor, en quien se sospechaba ruptura del saco, sin embargo, de manera intraoperatoria se encontró que la aparente ruptura del saco, correspondía a la comunicación con un divertículo de Meckel. El caso además se asoció con hallazgos ecocardiográficos de tetralogía de Fallot.

Palabras clave: onfalocele; divertículo de Meckel; tetralogía de Fallot; sutura mecánica.

\begin{abstract}
Omphalocele or exomphalos are defined as a congenital defect of the abdominal wall, which consists of the herniation of the abdominal viscera through the umbilical ring. This entity is rarely associated with the communication of Meckel's diverticulum with the omphalocele sac. Considering the low prevalence of this entity, we report the case of a newborn with a diagnosis of minor omphalocele, in whom rupture of the sac was suspected; however, intraoperatively it was found that the apparent rupture of the sac corresponded to a communication with a Meckel's diverticulum. The case was also associated with echocardiographic findings of tetralogy of Fallot.
\end{abstract}

Keywords: omphalocele; Meckel's diverticulum; tetralogy of Fallot; mechanical suture.

Fecha de recibido: 09/06/2020 - Fecha de aceptación: 21/08/2020 - Fecha de publicación en línea: 05/03/2021

Correspondencia: Álvaro Eduardo Sánchez-Hernández, Calle 141 \# 7B - 37, Apartamento 703, Bogotá. D.C., Colombia.

Teléfono: 316 6434929. Correo electrónico: appaloosa1991@gmail.com

Citar como: Sánchez-Hernández AE, Mogollón G, Luengas P, Del Rio S, Rotterman M, Mora N, Rueda S, Holguín DA, Cabrera LF, Rodríguez M, Piñeros S. Divertículo de Meckel en asociación a onfalocele y tetralogía de Fallot: Reporte de caso y revisión de la literatura. Rev Colomb Cir. 2021;36:334-7. https://doi.org/10.30944/20117582.634

Este es un artículo de acceso abierto bajo una Licencia Creative Commons - BY-NC-ND https://creativecommons.org/licenses/by-ncnd/4.0/deed.es 


\section{Introducción}

Se define onfalocele o exónfalos como un defecto congénito de la pared abdominal, que consiste en una herniación de las vísceras abdominales a través del anillo umbilical, cubierto por un saco compuesto principalmente por los mucopolisacáridos de la gelatina de Wharton. Tiene una incidencia aproximada de 1 por cada 4000 nacidos vivos, con una presentación variable, que puede llegar a ser gigante, como la describen algunos autores, en la cual hay compromiso del hígado.

En la literatura, por otra parte, se encuentran algunos casos de onfalocele o exónfalos, en asociación al divertículo de Meckel, con comunicación del divertículo en mención con el saco del onfalocele. Teniendo en cuenta la escasa prevalencia de esta asociación, presentamos en este artículo el caso de un paciente recién nacido, con diagnóstico de onfalocele menor y sospecha de ruptura del saco, con hallazgo intraoperatorio de divertículo de Meckel en comunicación con el saco, que correspondía a la aparente ruptura del saco.

\section{Presentación de caso}

Recién nacido por cesárea, con peso de 3,0 kg y masa que había sido diagnosticada previamente por ultrasonido obstétrico, protruida a nivel umbilical de $4 \mathrm{~cm}$, con aparente zona de ruptura a nivel del saco. El paciente fue llevado inmediatamente a cirugía para reducción del contenido herniario, pero al momento de practicar la incisión del saco, se encontró que la zona de ruptura correspondía a la llegada de un divertículo de Meckel (figuras 1 y 2), el cual se resecó con sutura mecánica lineal cortante $45 \mathrm{~mm}$ (figura 3) y finalmente se hizo el cierre de la pared abdominal. El paciente fue trasladado a la unidad de cuidado intensivo neonatal donde, mediante un estudio ecocardiográfico, se identificó una tetralogía de Fallot.

Luego de lograr la extubación y el retiro de los soportes vasoactivos, se remitió a un hospital de mayor complejidad para el manejo integral por parte del servicio de cirugía cardiovascular, quienes realizaron estudios de extensión, con el fin de definir el manejo quirúrgico definitivo en un plazo de seis meses.

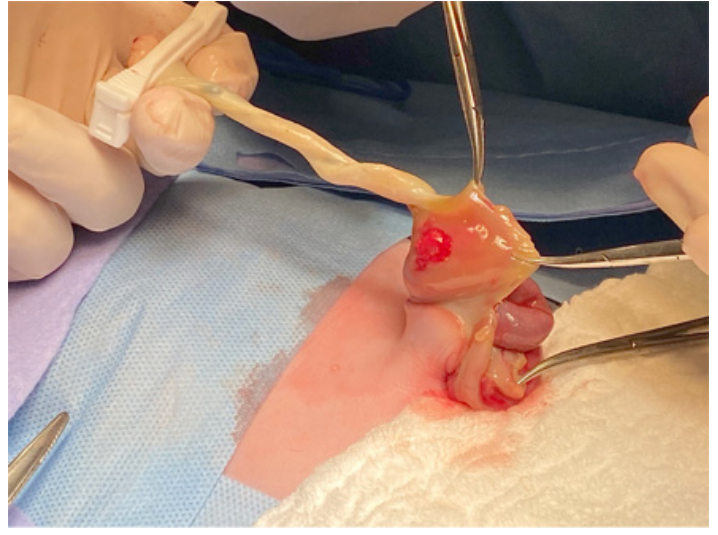

Figura 1. Comunicación del divertículo de Meckel con el onfalocele.

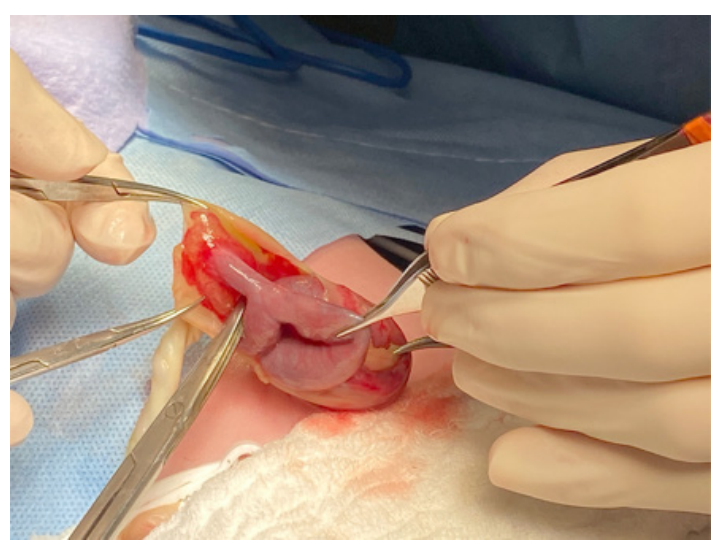

Figura 2. Al exteriorizar el contenido abdominal, se observa el divertículo de Meckel.

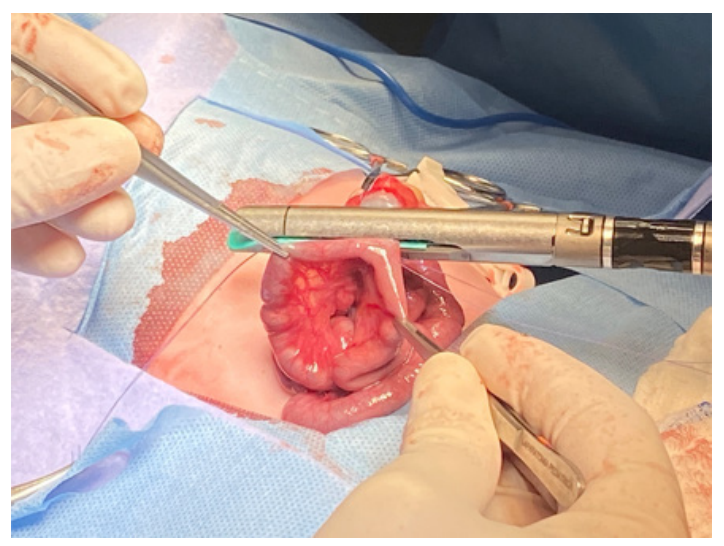

Figura 3. Resección del divertículo con sutura lineal cortante. 


\section{Discusión}

El onfalocele o exónfalo es uno de los defectos congénitos más comunes de la pared abdominal. Consiste en la herniación de las vísceras abdominales a través del anillo umbilical y puede tener una medida de 2 a $10 \mathrm{~cm}$. Se caracteriza porque el contenido herniario está cubierto por tres membranas, la capa amniótica externa, la capa media de Wharton y la capa peritoneal interna, lo que lo diferencia de otras patologías congénitas de la pared abdominal ${ }^{1}$. Los datos encontrados en la literatura proponen que aproximadamente un $80 \%$ pueden tener asociación a otra patología congénita, entre ellas cardiacas (10-40\%), gastrointestinales (3-20\%), genitourinarias (6-20\%) o del sistema nervioso central (3-20\%) $)^{1,2,3}$.

Con el desarrollo de la ultrasonografía, el diagnóstico de onfalocele y su asociación con otras patologías ha venido aumentando. Hace algunas décadas, el diagnóstico se realizaba solo al momento del nacimiento, mientras que, en la actualidad, es posible hacer el diagnóstico por imagen, incluso desde la semana 11 de gestación, dependiendo del compromiso de la pared abdominal y, por supuesto, de la habilidad del ecografista ${ }^{4,5}$.

La categorización del onfalocele según la presentación es epigástrica, umbilical o hipogástrica. Otros autores lo clasifican como menor o mayor, de acuerdo con el tamaño del defecto, y otros lo clasifican en pequeño, gigante o roto, dependiendo del tamaño del defecto mayor a 5 centímetros y del compromiso del hígado dentro de las vísceras contenidas en el saco ${ }^{1,3,5,6}$.

Otros métodos diagnósticos que tienen alguna utilidad son los niveles de alfa-fetoproteína materna, la acetilcolinesterasa en el líquido amniótico, la ecocardiografía fetal y los exámenes de cribado para patologías renales y musculoesqueléticas, sobre todo teniendo en cuenta la asociación a síndromes como trisomía 13, 18 y 21, pentalogía de Cantrell, ano imperforado, atresia anal, fístula traqueoesofágica, y atresia esofágica, entre otras. En cuanto al cariotipo, es de vital importancia tener en cuenta que los neonatos con cariotipo normal, pueden tener una supervivencia hasta del $95 \%{ }^{1,3,6}$.
Para el tratamiento definitivo, el cierre temprano de la pared abdominal, dependerá de la edad del paciente, al igual que del tamaño del defecto. Existen series de casos con seguimiento por seis años de pacientes con defectos menores a $1 \mathrm{~cm}$, que presentaron cierre espontáneo. No obstante, los defectos más grandes requieren tratamiento quirúrgico ${ }^{1,7,8}$, como en el paciente que presentamos, quien además tenía divertículo de Meckel y tetralogía de Fallot. Se deben diferenciar las situaciones asociadas a otras complicaciones, en particular en los casos de onfalocele, en que existe una alta probabilidad de malformaciones asociadas, por lo que se indica el estudio detallado de los pacientes ${ }^{9}$.

Por último, es importante tener en cuenta que los defectos de la pared abdominal han venido en aumento en la población latinoamericana ${ }^{10}$. Dada la complejidad de esta patología, algunos autores recomiendan el manejo multidisciplinario en centros de referencia ${ }^{11}$.

\section{Conclusión}

Lo mencionado en esta presentación a propósito del paciente tratado exitosamente, ratifica la importancia de la discusión y divulgación de estos casos, en el ámbito académico.

Agradecimientos: A la universidad por inculcar la labor investigativa en sus alumnos.

\section{Cumplimiento de normas éticas}

Consentimiento informado: Se obtuvo el consentimiento informado por parte de los padres del paciente.

Conflicto de intereses: Los autores no tienen potencial conflicto de interés con respecto a la publicación de este documento.

Financiamiento: Los autores afirman que no tuvieron ningún tipo de financiamiento para la realización de este trabajo.

\section{Contribución de los autores:}

Concepción y diseño del estudio: Álvaro Sánchez, German Mogollón, Pablo Luengas, Sergio Del Rio, Marcos Rotterman, Nicolás Mora, Sergio Rueda, Diana Alejandra Holguín, Luis Felipe Cabrera, Manuela Rodríguez, Santiago Piñeros. 
Adquisición de datos: Álvaro Sánchez, German Mogollón, Pablo Luengas, Sergio Del Rio, Marcos Rotterman, Nicolás Mora, Sergio Rueda, Diana Alejandra Holguín, Luis Felipe Cabrera, Manuela Rodríguez, Santiago Piñeros.

Análisis e interpretación de datos: Álvaro Sánchez, German Mogollón, Pablo Luengas, Sergio Del Rio, Marcos Rotterman, Nicolás Mora, Sergio Rueda, Diana Alejandra Holguín, Luis Felipe Cabrera, Manuela Rodríguez, Santiago Piñeros.

Redacción del manuscrito: Álvaro Sánchez, German Mogollón, Pablo Luengas, Sergio Del Rio, Marcos Rotterman, Nicolás Mora, Sergio Rueda, Diana Alejandra Holguín, Luis Felipe Cabrera, Manuela Rodríguez, Santiago Piñeros.

Revisión crítica: Álvaro Sánchez, German Mogollón, Pablo Luengas, Sergio Del Rio, Marcos Rotterman, Nicolás Mora, Sergio Rueda, Diana Alejandra Holguín, Luis Felipe Cabrera, Manuela Rodríguez, Santiago Piñeros.

\section{Referencias}

1. Puri P. Pediatric Surgery. Springer-Verlag GmbH Germany, part of Springer Nature 2020;1:65-82.

2. Kadian Y, Verma A, Rattan K, Kajal P. Vitellointestinal duct anomalies in infancy. J Neonatal Surg. 2016;5:30. https://doi.org/10.21699/jns.v5i3.351

3. Charki M, Abdellaoui H, Andaloussi S, Oukhouya M, Mahmoudi A, El madi A. et al. Congenital fistulisation of Meckel's diverticulum in omphalocele sac: case report. Pan Afr Med J. 2019;32:20

https://doi.org/10.11604/pamj.2019.32.20.15010
4. Gonzalez K, Chandler N. Ruptured omphalocele: diagnosis and management. Semin Pediatr Surg. 2019; 28:101-5.

https://doi.org/10.1053/j.sempedsurg.2019.04.009

5. Snyder, C. Current management of umbilical abnormalities and related anomalies. Semin Pediatr Surg. 2007;16:41-9.

https://doi.org/10.1053/j.sempedsurg.2006.10.006

6. Verla M, Style C, Olutoye O. Prenatal diagnosis and management of omphalocele. Semin Pediatr Surg. 2019;28:84-8.

https://doi.org/10.1053/j.sempedsurg.2019.04.007

7. Sohn H, Park K, Lee N, Kim M, Lee S. Meckel diverticulum in exomphalos minor. Ann Surg Treat Res. 2016;91:90. https://doi.org/10.4174/astr.2016.91.2.90

8. Mammadov E. Patent omphalomesenteric duct with protruding bowels through a ruptured omphalocele. Balkan Med J. 2018;35:118-9.

https://doi.org/10.4274/balkanmedj.2017.0230

9. Cuervo JL. Defectos de la pared abdominal. Rev. Hosp. Niños (B. Aires). 2015;57:170-190.

10. Barboza-Argüello M, Benavides-Lara A. Prevalencia al nacimiento de gastrosquisis y onfalocele en Costa Rica. Acta Méd Costarric. 2018;60:35-41.

11. Bertolotto A, Córdoba M, Vargas Vaca Y, Guzmán Cruz P, Álvarez A. Caracterización de los pacientes, tratamiento y complicaciones más frecuentes de los recién nacidos con gastrosquisis y onfalocele manejados en la Unidad de Recién Nacidos del Hospital Universitario San Ignacio. Experiencia de 10 años. Universitas Médica. 2017;57:323-331. 